\title{
Angélique, une série de « corsage et d'épée »
}

La figure d'Angélique est d'abord une création littéraire d'Anne Golon, une romancière très productive, qui imagine le personnage de la "marquise des Anges " en 1950. Son mari, Serge Golon, l'aide dans ses recherches documentaires, mais s'ils signent à deux leur saga éditée en France à partir de 1957 - et jusqu'en 1985 -, il semble qu'Anne Golon ait surtout rédigé seule ses romans. C'est d'ailleurs sous son seul nom que les ouvrages ont d'abord paru en Allemagne en 1956, et qu'ils sont de nouveau publiés, depuis $2009^{1}$, dans une édition revue et augmentée. Dans les années 1950, il devait encore être, en France, plus facile et vendeur qu'une œuvre de littérature populaire soit signée par un auteur masculin, ce qui explique la présence alors du nom des deux époux sur la couverture des romans d'Anne Golon. Plusieurs comptes rendus critiques de la série cinématographique firent d'ailleurs l'impasse sur la romancière et se contentèrent de citer Serge Golon comme unique auteur de l'œuvre'.

Incarnée à l'écran par Michèle Mercier, Angélique a donc la particularité d'être une héroïne créée par une femme. Ce personnage est doté de nombreuses qualités romanesques; la " marquise des Anges" se caractérise à la fois par sa beauté, son intelligence et son courage. Celle qui, pour se défendre, n'hésite pas à manier le couteau, et même le pistolet, comme un homme, reste avant tout une figure d'amoureuse. L'intrigue de la saga repose ainsi, en grande partie, sur la liaison passionnelle qu'elle entretient avec le

1. Aux éditions l'Archipel.

2. Notamment Henry Chapier, « Faut-il que l'argent soit antinomique du talent ? », Combat, 10 décembre 1964. 
comte Joffrey de Peyrac ${ }^{3}$, son mari, qui restera, malgré de nombreux rebondissements, son seul grand amour.

Un des scénaristes de la série cinématographique, Claude Brulé, a luimême défini les Angélique comme une histoire de "corsage et d'épée ${ }^{4}$ ", cette formule a le mérite de la clarté : les Angélique sont conçus comme des films historiques populaires destinés à un public habitué, depuis les années 1950 , aux films de cape et d'épée, et auquel on propose, dans ces années 1960 aux mœurs plus libres, une nouvelle combinaison : des films en costumes dans lesquels on sort encore l'épée, mais où une héroïne belle, désirable et peu farouche, s'est substituée au héros. Lors de la décennie précédente, Caroline chérie (Richard Pottier, 1951), adapté d'un roman de Cecil Saint-Laurent ${ }^{5}$, avait déjà lancé la mode des héroïnes historiques fictives gentiment volages, propulsant Martine Carol, l'interprète du rôle-titre, au rang de sex-symbol et de modèle de la féminité française. Noël Burch et Geneviève Sellier ${ }^{6}$ ont bien montré comment ce film, en dépit de ses apparences d'érotisme et de libertinage, respectait les conventions de l'époque. Si la jeune Caroline, avec ses multiples amants, semble représenter une femme émancipée, la fin du long métrage signale un retour à l'ordre masculin. Le personnage féminin reçoit de bonne grâce une gifle de la part de l'homme qu'elle aime, un coureur de jupons auquel, à l'inverse, il n'est fait aucun reproche. La série des Angélique s'inscrit dans la droite ligne de Caroline chérie ${ }^{7}$ : de l'écrit à l'écran, en effet, le personnage et l'univers narratif dans lequel il évolue vont connaître de nombreux changements à travers une relecture globalement conformiste, misogyne et érotisante. Ce mode de fonctionnement témoigne de l'orientation sexuée des représentations de la féminité élaborées et diffusées par le cinéma populaire français des années 1960. Nous nous proposons d'étudier les modalités de ce processus de transformation en montrant comment se croisent, dans l'adaptation audiovisuelle de cette série de littérature populaire, les regards d'une romancière d'un côté et d'hommes de cinéma de l'autre.

3. Interprété à l'écran par Robert Hossein, le personnage de Peyrac est laissé de côté dans l'intrigue littéraire pour que le récit soit focalisé sur Angélique. La romancière fait croire à ses lecteurs qu'après sa disgrâce - qui rappelle celle de Nicolas Fouquet - il a été brûlé pour sorcellerie en place de Grève, pour le ressusciter plus tard. Dans les romans, Joffrey est loin d'être le seul amant d'Angélique.

4. Comme le rapporte le critique Jean de Baroncelli dans sa recension du premier épisode de la série, Le Monde, 12 décembre 1964.

5. Pseudonyme de Jacques Laurent.

6. Noël Burch et Geneviève Sellier, La drôle de guerre des sexes du cinéma français (1930-1956), Paris, Nathan, 1996, p. 247.

7. La maquilleuse de Caroline chérie, Maguy Vernadet, sera également employée pour faire à Michèle Mercier l'œil de biche qu'avait popularisé Brigitte Bardot au début des années 1960. 
Très rapidement, les premiers volumes de la saga romanesque Angélique connaissent un immense succès public, non seulement en France, mais aussi en Italie et en Allemagne, et jusqu'en Union Soviétique et au Japon. Cette popularité suscite évidemment l'intérêt des producteurs de films qui comprennent très tôt le potentiel commercial du personnage. La première adaptation cinématographique des aventures d'Angélique entre ainsi en production six années après la publication en France du premier volume écrit par Anne Golon. Si le personnage d'Angélique a été inventé par une femme, le passage de l'héroïne au cinéma va considérablement transformer sa représentation. En effet, à l'époque, les films historiques à gros budget demeurent avant tout une affaire d'hommes : entre 1964 et 1968, les cinq épisodes ${ }^{8}$ sont ainsi réalisés par Bernard Borderie et produits par Francis Cosne. Tandis qu'Anne Golon a été mise à l'écart de l'adaptation, les scénaristes et dialoguistes qui la prennent en charge sont eux aussi exclusivement de sexe masculin : Claude Brulé, Daniel Boulanger, Pascal Jardin et Alain Decaux.

L'examen de la réception critique des cinq films qui composent la série révèle un accueil mitigé, voire sévère, lequel diffère très nettement des goûts d'un public populaire qui assure le succès dans les salles de chaque épisode. Les journalistes jugent avec mépris le travail du réalisateur Bernard Borderie, présenté comme un "Hunebelle du pauvre" ", et même relégué au rang de "régisseur de plateau ${ }^{10}$ " œuvrant dans des longs métrages décrits comme racoleurs et égrillards. Leur érotisme demeure pourtant bien sage, conformément aux limites que la censure de la $V^{e}$ République $^{11}$ fixe à un cinéma grand public qui doit se contenter d'aguicher sa clientèle ${ }^{12}$. Les qualificatifs employés pour relater les performances de Michèle Mercier ne sont pas plus tendres, on lui reproche de se dévêtir bien volontiers devant la caméra, et son interprétation est le plus souvent résumée à sa plastique ${ }^{13}$. Si la comédienne se

8. Angélique, marquise des Anges (1964), Merveilleuse Angélique (1965), Angélique et le Roy (1966), Indomptable Angélique (1967), Angélique et le Sultan (1968).

9. Titre de l'article de Michel Capdenac dans Les Lettres Françaises, 15 juillet 1965.

10. Henry Chapier, art. cité.

11. H. H., "Tous les charmes de l'érotisme Ve République, façon aventures retroussées historiques », Libération, 7 juillet 1980, à l'occasion d'une rediffusion télévisée du premier épisode.

12. "Aventures mouvementées que Michèle Mercier traverse en habituée des situations scabreuses, sachant montrer de son corps tout ce que la censure ne condamne pas ", Robert Chazal, France-Soir, 31 octobre 1967. « Un déshabillé vaporeux par-ci, une tenue de bain Louis XIV par-là. Cela passe très vite. II ne faut pas choquer le spectateur. Simplement lui prodiguer de petites excitations de bon aloi », ClaudeJean Philippe, Télérama, n̊841, 27 février 1966.

13. "Du sein. Beaucoup de seins », Jean-Louis Bory, Arts, n॰ 985, 16-22 décembre 1964. Sur l'exploitation des attraits physiques de l'actrice par la série se reporter à Sébastien Le Pajolec, " Michèle Mercier et Angélique : une séduction entre deux époques », dans Christian Delporte (avec Audrey Hermel), Corps et séduction, du charme à la manipulation, Paris, Éditions Nicolas Malais, 2013. 
déshabille souvent, livrant aux spectateurs de nombreuses vues de son décolleté, de son dos et même de ses fesses, elle n'apparaît pourtant jamais nue à l'écran dans la saga ${ }^{14}$ : son corps est soit occulté par un rideau vaporeux ${ }^{15}$, soit le cadrage s'arrête à la pointe de ses seins et de son nombril, l'actrice révélant plus tard avoir collé des sparadraps rouge et du plastique sur certaines parties de son corps parce qu'elle suspectait le réalisateur et le producteur de vouloir la montrer nue ${ }^{16}$.

La presse ne manque pas d'évoquer le caractère pervers voire sadomasochiste de plusieurs scènes de la série. Les adaptateurs ont ici consciencieusement repris du livre tous les passages de flagellation, nous donnant à voir des ribaudes dont on arrache le chemisier avant de se faire fouetter. Angélique défie la cravache et le fouet de son second mari, le violent Philippe de Plessis-Bellière, et se trouve elle-même fouettée dans le dernier épisode d'Angélique et le sultan ${ }^{17}$, un châtiment auquel elle avait réussi à échapper dans les épisodes précédents. À rebours de leur fidélité à l'écrit dès qu'il s'agit de retranscrire des scènes de soumission, les adaptateurs s'éloignent du modèle littéraire quand il est question de relater les rapports qu'Angélique entretient avec la gent masculine au cours de ses aventures. Si dans les romans d'Anne Golon, Angélique est dépeinte comme une héroïne éprise de liberté, qui sait ce qu'elle veut et ne se laisse pas faire, les films la métamorphosent en une jeune femme, belle et frivole, dont la conduite est le plus souvent dictée par les hommes. Cette relecture masculine et misogyne du personnage la rattache au modèle de la "ravissante idiote " popularisé quelques années plus tôt par Brigitte Bardot ${ }^{18}$. Les scénarios des cinq films, consultables à la Bibliothèque $\mathrm{du}$ film ${ }^{19}$, témoignent très nettement de cette déformation consciente

14. Comme elle prend soin de l'affirmer dans ses mémoires, dont elle a publié trois versions : Michèle Mercier, Angélique à cœur perdu, Paris, Carrère, 1987 ; Angéliquement vôtre, Paris, Lettres et Images, 1996 ; avec Henry-Jean Servat, Je ne suis pas Angélique, Paris, Denoël, 2002. En revanche, elle était apparue dénudée dans Tirez sur le pianiste (François Truffaut, 1961).

15. Lors d'un bref plan d'Indomptable Angélique sur le bateau du marquis d'Escrainville.

16. Michèle Mercier, Je ne suis pas Angélique, op. cit., p. 55-56.

17. Lire à ce sujet Christelle Taraud, "Angélique et l'Orient : une certaine vision de l'altérité ? ", L'Homme et la société, n¹54, 2004, p. 9-30. DOI : 10.3917//hs.154.0009.

18. Le rôle fut d'abord proposé à Brigitte Bardot qui le refusa sans avoir lu le scénario, ce qu'elle dira regretter par la suite. Roger Vadim, qui n'était déjà plus son mari à l'époque, lui avait parlé du projet pour lequel il avait écrit une scène : celle très suggestive où Robert Hossein caresse et met à nu une statue recouverte de terre devant sa femme très troublée. Une scène originale qui rajoute à l'érotisme du texte d'Anne Golon.

19. Scénarios d'Angélique marquise des Anges et de Merveilleuse Angélique, cote : SCEN 140 - B42 ; scénario d'Angélique et le Roy, cote : SCEN 139 - B41; scénario d'Indomptable Angélique et d'Angélique et le Sultan, cote : SCEN 1403 - B415. 
du personnage qui porte la trace d'un regard masculin : on y lit, par exemple, qu'Angélique " exhibe son anatomie supérieure ${ }^{20}$ " dans des " révérences qui mettent en valeur la beauté de son décolleté21 ». Si Anne Golon a décrit une femme belle et désirable, à aucun moment elle n'a imaginé que sa révérence puisse être un motif d'excitation masculine.

Les scénaristes transforment de manière particulièrement sensible les caractéristiques de l'héroïne de papier dès qu'il s'agit de relater ses aventures sexuelles. En effet, les épisodes cinématographiques la montrent très souvent au lit en charmante compagnie masculine, répartissant ses partenaires sentimentaux en deux catégories. La première regroupe ses époux légitimes - Joffrey de Peyrac puis Philippe du Plessis - avec lesquels la morale est sauve, ce qui permettra de focaliser les scènes sur l'érotisation du corps de Michèle Mercier. Le second ensemble tend à présenter la " marquise des Anges " comme une collectionneuse d'amants - le bandit Calembredaine, le Poète Crotté et le Prince Racoczi principalement. Ces scènes privilégient souvent les protagonistes masculins (Giuliano Gemma, Jean-Louis Trintignant et Fred Williams) en exhibant leurs torses devant une Angélique qui garde ses vêtements. Le personnage féminin n'est plus ici au centre de l'action, le regard s'est déporté sur ses amants, comme en attestent les descriptions présentes dans les archives scénaristiques : "le poète qui vient de sortir avec, pour tout vêtement une serviette nouée autour des reins "; "Le drap à demi baissé nous fait comprendre qu'il est nu dans le lit "; " assis devant la coiffeuse, torse nu, Racoczi se fait couper les cheveux par Angélique, qui est en chemise de nuit $»^{22}$.

Deux étreintes, détaillées dans les romans, ne sont plus que suggérées dans l'adaptation cinématographique. La première relate un viol dont Angélique est victime alors qu'elle est venue plaider, au Louvre, la cause de son premier mari : un homme l'assomme et, tandis qu'il l'allonge sur un divan, le plan est coupé ; nous n'en verrons pas plus, comme l'indiquait déjà le scénario $^{23}$. La seconde concerne une aventure d'une nuit avec un capitaine de police. Dans le texte d'Anne Golon l'héroïne consentait à coucher avec un gros capitaine aux manières frustes afin d'échapper au fouet et de sauver ses enfants. Le dégoût des premiers instants se transformait finalement en un moment de plaisir. Cette scène est radicalement modifiée à l'écran par l'élimination de

20. «Tous les charmes de l'érotisme Ve République, façon aventures retroussées historiques », art. cité.

21. Scénario d'Angélique marquise des Anges, op. cit.

22. Scénario de Merveilleuse Angélique, op. cit.

23. «Vardes réussit à bloquer les poignets d'Angélique dans une seule main et, de l'autre, la gifle à toute volée. La tête d'Angélique rebondit contre le mur : elle tombe évanouie. Vardes la couche sur la banquette. Vardes dénude les épaules d'Angélique et les lui embrasse avec avidité. Angélique est comme morte ». 
l'acte sexuel et son remplacement par un gag, substituant le trivial au bestial. Devant la caméra de Bernard Borderie on voit bien le gros capitaine essayer d'attraper Angélique, mais celle-ci se débat et l'assomme, et, lorsqu'un sergent vient pour l'arrêter pensant qu'elle vient de tuer son supérieur, ce dernier se met à ronfler bruyamment, ce qui déclenche le rire des deux personnages. Les modifications que comportent ces deux séquences résultent sans doute de leur inadéquation dans le cadre de la transposition audiovisuelle du matériau d'origine ; en effet, les émotions érotiques qu'elles étaient susceptibles de produire auprès des spectateurs ne correspondaient probablement pas aux rapports entre les sexes tels que les productions cinématographiques hexagonales de la période les définissaient. Dans leurs adaptations, les scénaristes accordent ainsi une place privilégiée aux ébats mutuellement consentis. De ce point de vue ils répondent aux exigences du cinéma populaire français des années 1960 : les films proposent un érotisme sage dans lequel (surtout) Angélique, à la fois fidèle et volage, se plie " avec plaisir " aux désirs de " certains " hommes.

Ainsi, alors que les romans pouvaient procurer à l'héroïne des aventures amoureuses ambiguës, les longs métrages les simplifient et les normalisent au service d'une relecture misogyne d'un personnage désormais soumis aux conventions morales les plus traditionnelles. Sous la plume d'Anne Golon, Angélique apparaît comme une femme décidée à suivre ses envies sans forcément tenir compte des usages de son temps, tandis qu'au cinéma elle est représentée comme beaucoup plus hésitante à enfreindre ces règles. La séquence avec son ami d'enfance, Nicolas, au début du récit s'avère exemplaire de la manière dont le travail du cinéaste dénature le discours de la romancière. Avant de partir retrouver un mari auquel on l'a vendue et qu'elle imagine répugnant, Angélique veut décider de l'homme auquel elle se donnera pour la première fois, elle choisit Nicolas et l'attire dans la grange. En dépit de la brutalité du jeune homme elle se laisse faire, et l'étreinte n'est interrompue que suite à l'arrivée inopinée de sa tante et d'un valet.

De toute sa volonté, elle voulait que ce fût fait et vite, car on pouvait les surprendre. Les dents serrées, elle se répétait que ce ne serait pas l'autre qui la prendrait le premier. Ce serait la réponse jetée à l'or, qui croyait pouvoir tout acheter. Elle voulait, elle voulait vraiment que cet acte s'accomplisse. Mais à plusieurs reprises, elle se retint de repousser ce corps pesant dont une soudaine folie s'emparait. Il y eut un brusque éclat de lanterne à travers la grange et, de la porte, un cri de femme horrifiée s'éleva ${ }^{24}$. 
Bernard Borderie filme, lui, une Angélique qui paraît beaucoup moins sûre d'elle-même et nettement plus morale. Si elle attire toujours Nicolas dans la grange, elle commence à se débattre dès qu'il la serre contre lui, et lui demande d'arrêter. Elle sera finalement délivrée par l'arrivée du valet. De l'écrit à l'écran l'audacieuse détermination s'est muée en un agaçant caprice.

Lorsque l'on confronte les caractéristiques d'Angélique telles que les élaborent les romans à celles définies dans les scénarios puis montrées dans les films, il apparaît que les processus de transformation du personnage que nous avons identifiés sont, en fait, progressifs. En effet, la consultation des scénarios indique que les adaptateurs n'avaient pas renoncé, du moins en partie, à dépeindre la "marquise des Anges" comme une femme d'action. Ainsi, dans le script du premier long métrage de la série, après que le comte de Peyrac a tué de sa lame le chevalier de Germontaz qui avait insulté Angélique ${ }^{25}$ cette dernière proclame à son mari : «maintenant, s'ils te lynchent, ils auront affaire à moi ». Quand Peyrac l'interroge : "tu prendrais l'épée ? ", elle conclut avec détermination : "il faudra que tu m’apprennes ». La version filmée supprime ces dialogues dans lesquels transparaissaient encore les qualités d'une véritable héroïne "de corsage et d'épée ", leur préférant un échange plus convenu où triomphent l'amour et le désir. La comparaison entre les romans et les scénarios démontre néanmoins que le personnage connaît une véritable évolution, notamment dans son rapport à la sexualité, dès le travail d'adaptation.

L'examen des indications présentes dans les scripts pour orienter le jeu de la comédienne nous renseigne sur les distances prises avec un matériau littéraire dans lequel Anne Golon sexualisait nettement plus l'héroïne. La première séquence du récit propose ainsi un échange teinté d'un certain érotisme entre une Angélique adolescente et son ami Nicolas, mais les didascalies, fidèlement suivies par le film, dessinent le portrait d'une jeune fille un peu niaise :

Angélique, au lieu de fuir, se précipite sur Nicolas et l'asperge, elle aussi. Quand elle se rapproche, on voit se dessiner, sous le corsage trempé qui colle à sa peau, les formes adorables de sa jeune poitrine. Angélique ne s'est pas aperçue du trouble de Nicolas et continue à l'asperger. Dans son agitation et dans ses gestes, les formes de son corsage se font de plus en plus précises. Son expression grave étonne Angélique, qui n’est pas consciente de sa semi-nudité.

Elle n'a pas conscience de sa sensualité, qu'à l'inverse Nicolas et les spectateurs, eux, perçoivent, notamment lorsqu'elle se protège le visage de ses bras

25. Dans la version d'Anne Golon, il avait attenté à son honneur en l'embrassant de force, tandis qu'à l'écran il ne s'agit que d'une simple insulte. 
et que leurs yeux devinent sa poitrine sous son corsage mouillé. La conscience de soi et la relative autonomie dont le personnage est doté dans les romans d'Anne Golon subissent la même remise en cause au début du deuxième long métrage de la série, alors qu'Angélique a tout perdu (mari, biens, rang). Elle est recueillie par des brigands parisiens dont le chef Calembredaine - qui n'est autre que Nicolas - la convoite et finit par obtenir d'elle ce qu'il désire. Tandis que dans le livre Angélique résistait longuement avant de se résoudre à lui céder, à l'écran toute forme de lutte a disparu.

Lui : " Méfie-toi. Je peux te prendre de force, si je veux ».

Elle : «Essaie un peu, je t’arracherai les yeux avec mes ongles ${ }^{26}$.

S’il n'y avait ce « je te déteste " prononcé avec peu de conviction par la jeune femme, et auquel succède, après un montage $c u t$, un " c'est moi que je déteste " qui déplace la culpabilité du côté de la femme, l'héroöne pourrait ici sembler parfaitement consentante. D'autant que la mise en scène, la montrant bras nus et presque satisfaite, laisse peu de place à l'ambiguïté.

On observe enfin le même glissement - de la femme sujet qui résiste à la femme objet qui se soumet - dans le passage concernant la relation entretenue par l'héroïne et son second mari, son cousin Philippe de Plessis-Bellière. La version littéraire suggère une Angélique calculatrice qui se rapproche de son cousin, décrit comme un homme odieux et dur avec les femmes, pour le faire chanter et ainsi l'épouser afin de retrouver son rang. Ce nouveau mari la déteste et la viole lors de leur nuit de noces. Le long métrage narre une toute autre histoire dans laquelle Angélique est d'abord la maîtresse de Philippe, puis renonce à le faire chanter avant de l'épouser. Si, dans l'adaptation cinématographique, ce dernier peut aussi se comporter violemment, il y est, à l'inverse du roman, paré de certaines qualités, et Angélique prend de toute évidence beaucoup de plaisir dans ses bras. Anne Golon avait imaginé une relation brutale et sombre entre ces deux personnages, mais Bernard Borderie, lui, en propose une mise en images atténuée où point même une dimension quasi romantique, très éloignée du propos original.

$\mathrm{La}$ « marquise des Anges » qui remplit les salles de cinéma s'avère donc être un personnage bien différent de l'Angélique qui triomphe dans les librairies. Selon qu'elle évolue sous le regard de la romancière ou des hommes de cinéma, cette figure promeut des féminités distinctes, parfois même contradictoires,

26. Anne et Serge Golon, Angélique, Le Chemin de Versailles 1, Paris, J'ai lu, 1976, p. 55. 
ce dont Anne Golon s'est d'ailleurs plaint à plusieurs reprises ${ }^{27}$. Elle avait créé le portrait original d'une femme à la fois libre et amoureuse, passionnée et tenace, parfois presque émancipée. Une héroïne d'un siècle passé susceptible d'annoncer des temps à venir. Le rôle interprété par Michèle Mercier propose une incarnation beaucoup moins novatrice, presque passéiste, en dépit d'un érotisme à la mode sixties: une femme qui aime faire l'amour, choisit ses amants de façon souvent capricieuse et se soumet volontiers au désir masculin exprimé à travers les yeux de ses partenaires et l'objectif de la caméra. Dans le sillage des interprétations à succès de Martine Carol et de Brigitte Bardot ${ }^{28}$ lors de la décennie précédente, Angélique, revue et corrigée par un cinéma français dominé par des hommes, présente une nouvelle "femme-objet "luxueuse"29 " dont le clinquant de la superproduction historique ne parvient pas à masquer la soumission.

27. Dans un entretien, accessible en ligne : http://www.radio.cz/fr/rubrique/miroir/un-accueil-chaleureuxpour-anne-golon-a-prague, la fille d'Anne Golon qui est aussi son éditrice, Nadia Goloubinoff, condense les critiques de la romancière à l'encontre du film : "Pour ceux qui n'ont pas lu le livre, c'est un film agréable à voir et, finalement, pourquoi pas. Mais quand on a lu le livre, on veut autre chose. Les femmes et même les hommes qui ont aujourd'hui des rapports plus normaux qu'auparavant avec les femmes, qui aiment les femmes intelligentes, les femmes qui ont de l'esprit, et non pas tellement une femme capricieuse qui dit oui et après qui dit non, il y en a comme ça, mais ce n'est pas la majorité des femmes. Et Angélique est une femme normale. Ce n'est pas bien sûr une chercheuse en biologie, mais elle n'a rien à voir avec le personnage du film. La femme dans le film est jolie, mais capricieuse, qui se débat comme elle peut, mais qui a des crises de nerfs, qui tue. Tandis que dans le livre, elle tue seulement pour sauver ses enfants. Vous voyez, il y a beaucoup de différence dans le caractère entre le film et le livre. »

28. Bien avant Angélique, le personnage de Juliette, joué par Brigitte Bardot dans Et Dieu créa la femme (Roger Vadim, 1956), avait revendiqué une forme de liberté sexuelle. Mais, comme l'ont montré Noël Burch et Geneviève Sellier (La drôle de guerre..., op. cit., p. 276), son émancipation ne posait guère de problème puisqu'elle restait un personnage assujetti au désir masculin.

29. Jacques Siclier, « Ça suffit, Madame la marquise », Télérama, n 930, 12 novembre 1967. 